\title{
EVALUACIÓN MICROBIOLÓGICA DE UN CUERPO DE AGUA DEL ACR HUMEDALES DE VENTANILLA (CALLAO, PERÚ) Y SU IMPORTANCIA PARA LA SALUD PÚBLICA LOCAL
}

\section{MICROBIOLOGICAL EVALUATION OF A WATER BODY AT ACR VENTANILLA WETLANDS (CALLAO, PERU) AND ITS RELEVANCE TO LOCAL PUBLIC HEALTH}

\author{
Roxana Rodríguez ${ }^{1}$, Rocío Retamozo-Chavez ${ }^{1,2}$, Héctor Aponte ${ }^{3,4}$ y Elfer Valdivia ${ }^{5,6}$
}

\section{Resumen}

Los humedales son ecosistemas muy importantes en la costa central del Perú. Muchos de estos ambientes están afectados por las poblaciones humanas, quienes también hacen uso de los mismos con fines recreativos. Con el objetivo de realizar un diagnóstico microbiológico de los cuerpos de agua del Área de Conservación Regional (ACR) Humedales de Ventanilla, donde las poblaciones aledañas realizan actividades recreativas, se realizaron muestreos mensuales entre agosto 2014 a julio 2015. En las muestras colectadas se cuantificaron coliformes totales, coliformes termotolerantes, Escherichia coli, y Enterococcus faecalis. Asimismo, se determinó de manera cualitativa la presencia o ausencia de Vibrio spp. y Salmonella spp. en cuatro estaciones donde la población tiene mayor acceso. Para la obtención de los límites máximos permisibles (LMP) se utilizaron los Estándares peruanos de Calidad Ambiental para el Agua (ECA) del año 2008. De las 15 estaciones monitoreadas, seis de ellas presentaron un promedio anual de coliformes totales mayor al LMP (>2000 NMP/100 mL), y para el caso de los coliformes termotolerantes, dos estaciones sobrepasaron los LMP (>1000 NMP/100 mL). La presencia de Vibrio spp. ha sido casi constante en tres de las cuatro estaciones evaluadas. Salmonella spp. estuvo ausente en todos los puntos. Los resultados evidencian que las actividades humanas están alterando los cuerpos de agua, pudiendo convertirlos en un reservorio de microorganismos patógenos para la salud de la población que lo utiliza.

Palabras clave: Contaminación del agua, coliformes totales, coliformes termotolerantes, humedales, límite máximo permisible (LMP).

\begin{abstract}
Wetlands are very important ecosystems in the central coast of Peru. Many of these environments are affected by human populations, who also make use of them for recreational purposes. With the aim of making a microbiological diagnosis of one of the water bodies of the Regional Conservation Area (ACR) Wetlands of Ventanilla, where the surrounding population perform recreational activities, a monthly monitoring program was conducted from august 2014 to July 2015, using 15 monitoring stations. The quantity of total coliforms, thermotolerant coliforms, Escherichia coli, Enterococcus faecalis was determined. The presence or absence of Vibrio spp. and Salmonella spp. in four stations where the population has more access was also determined. To obtain the maximum permissible limits (MPL), the 2008 Peruvian Environmental Quality Standards for Water (ECA) were used. Of the 15 monitored stations, 6 had an annual average of Total Coliforms greater than the MLP (> $2000 \mathrm{MNP} / 100 \mathrm{~mL}$ ), and 2 stations exceeded the MLP for thermotolerant coliforms ( $>1000 \mathrm{MNP} / 100 \mathrm{~mL}$ ). The presence of Vibrio spp. has been constant in three of the four stations evaluated. Salmonella spp. was absent in all points. The results show that human activities are altering this water body and can turn it into a reservoir of pathogenic microorganisms to human health; especially for the surrounding population that uses this small lake as a recreational area.
\end{abstract}

Key words: fecal coliforms, maximum permissible limit (MPL), total coliforms, water pollution, Wetlands. 


\section{Introducción.}

Los humedales de la costa de Lima y Callao son ecosistemas muy importantes dado que sirven de refugio para una gran cantidad de especies en medio del desierto peruano (Iannacone et al., 2010; Aponte \& Cano, 2013). Asimismo, provee de múltiples bienes y servicios ecosistémicos a las poblaciones humanas cercanas, como la provisión de fibras y de un paisaje adecuado para realizar actividades turísticas (León et al., 1998; Riveros et al., 1998). Geográficamente, estos ecosistemas se encuentran rodeados de la urbe más abundante del país, lo que los convierte en ecosistemas altamente vulnerables a diferentes actividades humanas inadecuadas que se desarrollan en sus inmediaciones como el desecho de basura, uso de sus lagunas como silos, incendios, entre otros (Young, 1998; Aponte et al., 2015).

Uno de estos ecosistemas es el Área de Conservación Regional (ACR) Humedales de Ventanilla. Este ecosistema es un espacio de aproximadamente 275 hectáreas destinadas a la protección de la vida silvestre ubicado en la Provincia Constitucional del Callao (Perú). Este ecosistema está compuesto por gramadales, juncales y salicorniales así como de cuerpos de agua que son hábitat de una gran diversidad de organismos (Alvarez \& Iannacone, 2008; Aponte \& Ramirez, 2014). Las lagunas que conforman este ecosistema albergan también setenta especies de protozoarios de vida libre, entre las que se encuentran especies bacterívoras (como Aspidisca cicada O. F. Müller, 1786; Aspidisca lynceus O.F. Müller, 1773, Cristigera phoenix Pennard, 1922; Cristigera setosa Kahl, 1928; Cyclidium glaucoma O.F. Müller, 1786 y Vorticella convallaria L., 1767), las cuales indican la probable contaminación de dichos cuerpos de agua (Guillén et al., 2015). Esta situación es preocupante, dado que parte de estas lagunas son utilizadas por la población con fines recreativos, sobre todo en los meses de verano. Hacer el seguimiento de otros indicadores de contaminación, como son la presencia de enterobacterias (coliformes totales $y$ coliformes termotolerantes), es fundamental para comprender el peligro real al cual puede estar expuesta la población de esta localidad.

El presente trabajo tiene como objetivo realizar un diagnóstico microbiológico en los cuerpos de agua usados por la población que vive en los alrededores del ACR Humedales de Ventanilla (Callao, Perú).

Area de estudio.

Los Humedales de Ventanilla se encuentran en la Provincia Constitucional del Callao, Distrito de Ventanilla, junto a la Playa Costa Azul, entre el Asentamiento Humano Defensores de la Patria y la Nueva Ciudadela Pachacútec (Figura 1 a y b). Los Humedales de Ventanilla limitan por el sur con colinas bajas, características del inicio de la Cordillera de los Andes; al este, colinda con zonas de expansión urbana como el AA. HH. Defensores de la Patria; y al oeste, con la línea paralela al mar. Alrededor existen
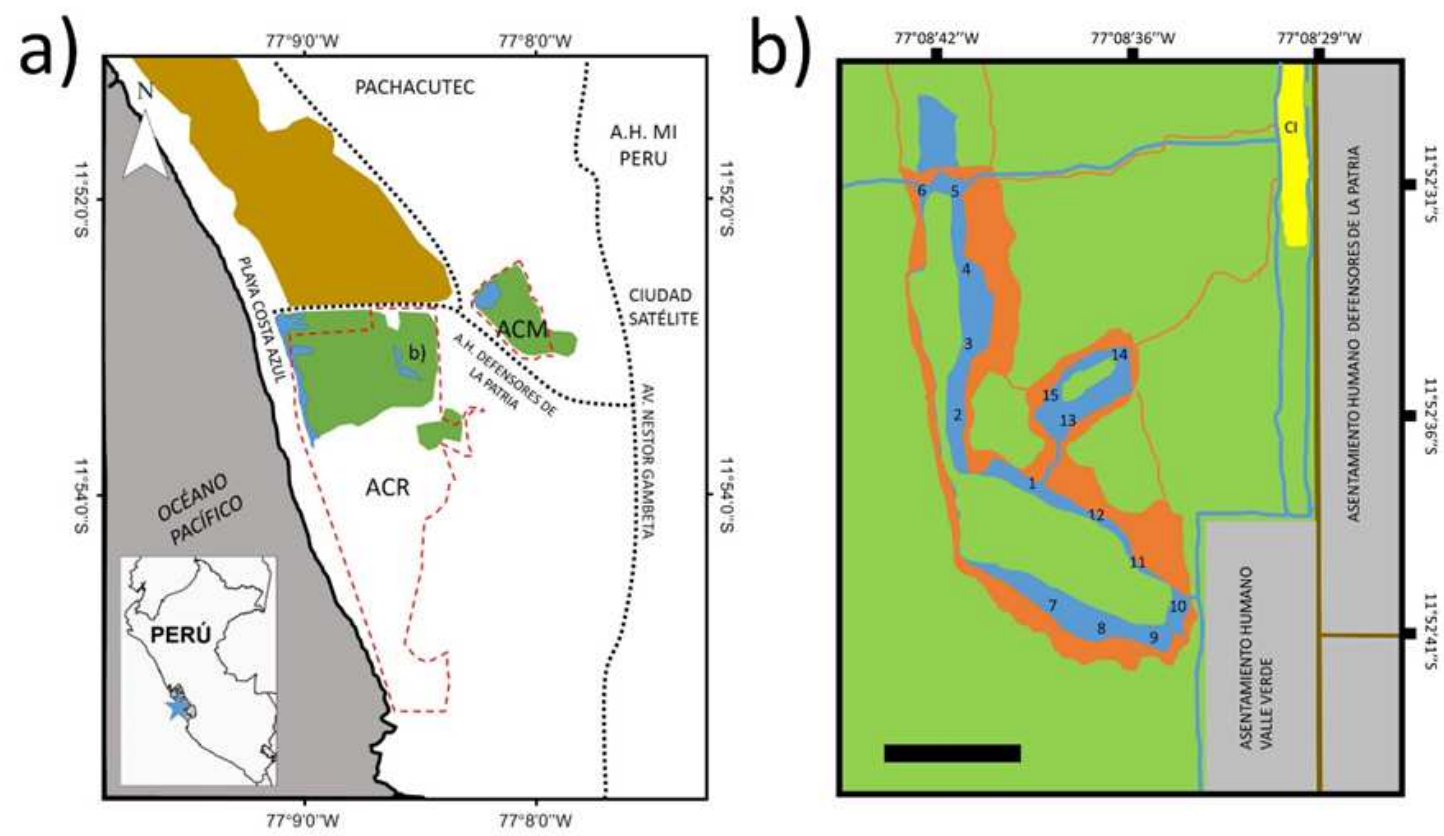

Figura 1. Área de Estudio. a) Ubicación del ACR Humedales de Ventanilla en el Perú y situación geográfica. $\mathrm{ACR}=$ Área de Conservación Regional; $\mathrm{ACM}=$ Área de Conservación Municipal. Los límites de ambas áreas se muestran con las líneas punteadas. b) Detalle del área de estudio y la laguna analizada. 1-15=Estaciones de muestreo. $\square=$ =egetación de Humedales; $\square=$ =Zona de tránsito alrededor de las lagunas; $\square=$ Cuerpos de agua y canales; =Centro de Interpretación; $\square=$ =elicto de humedal de uso agrícola. 
zonas con vegetación de humedales que no están protegidas y que están destinadas a actividades agrícolas, ganaderas y de construcción (Aponte \& Ramirez, 2014).

Parte de este ecosistema es protegido desde el año 2006 por el Gobierno Regional del Callao (Decreto Supremo No 074-2006-AG) con la categoría de Área de Conservación Regional (ACR). El ACR Humedales de Ventanilla cuenta con una superficie de 275.45 ha $y$ su acuífero es alimentado por infiltraciones marinas y por agua dulce procedente de la Cuenca del Rio Chillón (Rojas-Rieckhof, 2010). El presente estudio se realizó únicamente en el área comprendida dentro del ACR.

\section{Materiales y métodos.}

Recolección y procesamiento de las muestras.

El monitoreo se realizó mensualmente desde agosto del 2014 a julio del 2015 en la laguna denominada "Pisciplaya", laguna que es utilizada para recreación por la población circundante, en especial en los meses de verano, cuando centenas de pobladores la visitan (Gobierno Regional del Callao, 2009). Para determinar la calidad microbiológica del agua, se establecieron 15 estaciones de monitoreo localizadas en la periferia de la laguna (Figura 2). En estas estaciones se tomaron muestras de agua superficial. En ellas se cuantificaron los coliformes totales y termotolerantes, según lo indicado por el ECA del Perú (D.S. N002-2008-MINAM. 2008). La población circundante tiene mayor acceso a cuatro de estos puntos (1, 5, 10 y 15); en ellos, además de lo indicado previamente, se cuantificó Escherichia coli, y
Enterococcus faecalis, y se evaluó la presencia de Vibrio spp. y Salmonella spp. por ser patógenos de interés sanitario.

Muestreo y procesamiento de parámetros microbiológicos.

El muestreo y el procesamiento de todos los parámetros microbiológicos se realizaron teniendo en cuenta las recomendaciones y métodos estándar descritos por Rice y colaboradores (Rice et al., 2012). Para cuantificar coliformes totales, coliformes termotolerantes, Escherichia coli y Enterococcus faecalis se utilizaron los métodos SM-9221B, SM9221E, SM-9221F y SM 9230-SM 9230.B respectivamente. La lectura se hizo utilizando la tabla NMP (SM-9221B). Para la determinación de la presencia o ausencia de Vibrio spp. y Salmonella spp. se utilizaron los métodos SM 9260.B y SM 9260.H.

Análisis de Resultados.

Los resultados de los puntos 1, 5, 10 y 15 fueron comparados con los límites máximos permitidos (LMP) de la categoría 1B1 (Aguas superficiales destinadas para recreación de contacto primario) determinado por los Estándares de Calidad Ambiental (ECA) del Perú (D.S. Nº02-2008-MINAM, 2008). Durante el presente monitoreo se aprobó una nueva versión de los ECAs (Decreto Supremo No 015-2015MINAM) en el cual se retira el parámetro coliformes totales; sin embargo lo hemos mantenido ya que la presencia de coliformes totales nos permite establecer el grado de degradación de los cuerpos agua y es utilizado internacionalmente como indicador de contaminación (Pullés, 2014).

El resto de los puntos se compararon con los LMP a)

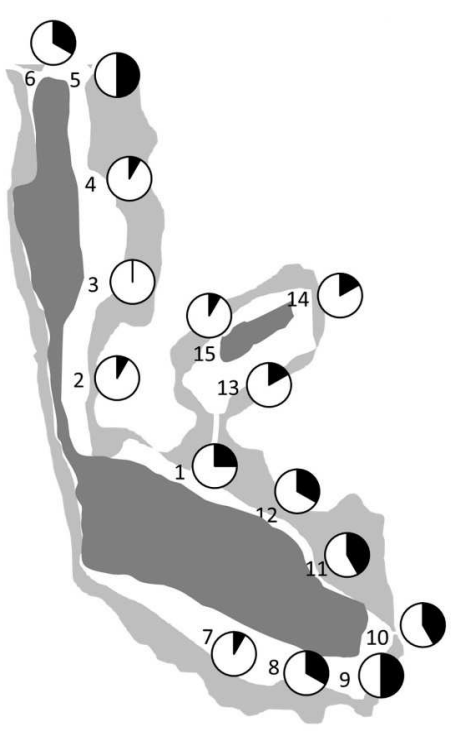

b)

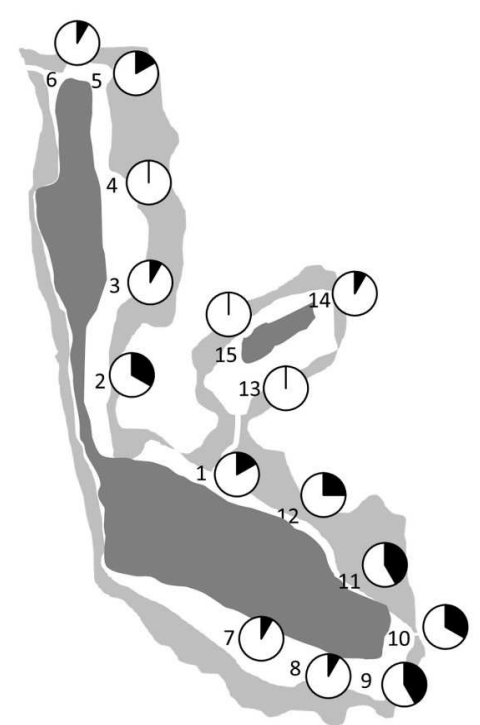

Figura 2. Frecuencia en que sobrepasó el ECA para coliformes totales (a) y termotolerantes (b) durante el presente estudio. La frecuencia está representadas por gráficos de pastel al lado de cada punto de muestreo con color negro. 
Tabla 1. Concentración bacteriana de coliformes totales (NMP/100 mL) en la laguna "Pisciplaya" por punto de muestreo. Los valores sombreados corresponden a los valores que sobrepasan los LMP).

\begin{tabular}{|c|c|c|c|c|c|c|c|c|c|c|c|c|c|c|c|}
\hline \multirow[b]{2}{*}{ Mes } & \multicolumn{15}{|c|}{ Puntos de muestreo } \\
\hline & 1 & 2 & 3 & 4 & 5 & 6 & 7 & 8 & 9 & 10 & 11 & 12 & 13 & 14 & 15 \\
\hline Agosto & 1700 & 790 & 700 & 5400 & 24000 & 3500 & 270 & 2400 & 35000 & 92000 & 9200 & 9200 & 68 & 330 & 170 \\
\hline Septiembre & 3500 & 1300 & 1700 & 230 & 3500 & 3500 & 490 & 220 & 3500 & 5400 & 2400 & 2200 & 3300 & 780 & 490 \\
\hline Octubre & 490 & 9200 & 1300 & 330 & 330 & 130 & 490 & 2400 & 2400 & 3500 & 1100 & 790 & 220 & 45 & 110 \\
\hline Noviembre & 45 & 460 & 170 & 20 & 130 & 130 & 20 & 170 & 210 & 140 & 230 & 220 & 20 & 68 & $<1.80$ \\
\hline Diciembre & 5400 & 490 & 1300 & 230 & 9200 & 1700 & 20 & 170 & 210 & 330 & 230 & 220 & 330 & 9200 & 110 \\
\hline Enero & 1300 & 1700 & 1700 & 790 & 3500 & 3500 & 790 & 210 & 410 & 5400 & & 1100 & 2400 & 490 & 2400 \\
\hline Febrero & 1700 & 1300 & 20 & 130 & 120 & 1700 & 170 & 790 & 5400 & 2200 & 790 & 1300 & 170 & 210 & 130 \\
\hline Marzo & 700 & 340 & 20 & 230 & 260 & 790 & 130 & 790 & 2200 & 1700 & 790 & 1700 & 330 & 210 & 110 \\
\hline Abril & 310 & 240 & 20 & 330 & 210 & 490 & 170 & 490 & 940 & 700 & 330 & 1100 & 230 & 170 & 140 \\
\hline Mayo & 170 & 45 & 20 & 110 & 45 & 220 & 790 & 2200 & 490 & 1100 & 5400 & 9200 & 220 & 1100 & 140 \\
\hline Junio & 9200 & 1200 & 700 & 340 & 9200 & 1700 & 490 & 170 & 340 & 330 & 1300 & 790 & 330 & 9200 & 110 \\
\hline Julio & 240 & 1300 & 790 & 230 & 5400 & 3500 & 9200 & 9200 & & 9200 & 9200 & 9200 & 1300 & 490 & 790 \\
\hline PROMEDIO & 2062.9 & 1530.4 & 703.3 & 697.5 & 4657.9 & 1738.3 & 1085.8 & 1600.8 & 4550.0 & 10166.7 & 2764.2 & 3085.0 & 743.2 & 1857.8 & 391.7 \\
\hline
\end{tabular}

Valor de referencia de LMP para coliformes totales $=2000 \mathrm{NMP} / 100 \mathrm{~mL}$ (ECAs, 2008).

de la Categoría 4 (Conservación del Ambiente Acuático) para la determinación del grado de conservación, dado que las lagunas no tienen un fin recreativo declarado, sino más bien la conservación de este ecosistema y de las especies que en ellos viven (Gobierno Regional del Callao, 2009). Se hicieron gráficos que representasen la frecuencia en que se sobrepasó el límite de los ECAs.

\section{Resultados.}

Durante el período de muestreo la concentración de coliformes totales excedió el LMP por lo menos en un mes en todos los puntos muestreados salvo en el punto 3 donde no se sobrepasó el LMP en ningún mes (Tabla 1, Figura 2a). Las estaciones 5, 9 y 10 obtuvieron los valores más altos durante el monitoreo (24000 MNP/100 mL, $35000 \mathrm{MNP} / 100 \mathrm{~mL}$ y 92000 $\mathrm{MNP} / 100 \mathrm{~mL}$ respectivamente en el mes de agosto). Los puntos 1, 5, 9, 10, 11 y 12 presentaron un promedio anual mayor al LMP, siendo el punto 10 el que presenta el promedio anual más alto (10167 NMP/100 mL).

La concentración de coliformes termotolerantes en los puntos 4, 6, 13 y 15 no excedieron el LMP; las estaciones restantes presentaron valores que superaron dicho límite en al menos un mes (Tabla 2, Figura 2b). Los puntos 2, 8 y 11 obtuvieron los valores más altos (9200 MNP/100 mL, $5400 \mathrm{MNP} / 100 \mathrm{~mL}$ y 5400 $\mathrm{MNP} / 100 \mathrm{~mL}$ respectivamente). El promedio anual de los puntos 2 y 11 estuvo por encima del LMP (1232
NMP/100 mL y 1237 NMP/100 mL respectivamente).

Se observó una fuerte presencia de Escherichia coli y Enterococcus faecalis en el punto 10. Este punto presentó los valores más altos entre las estaciones para ambos indicadores (Tablas 3 y 4); en los otros puntos la presencia fue menor. La presencia de Vibrio spp. fue casi constante durante el período de monitoreo, estando presente en todos los puntos (Tabla 5). Salmonella spp. estuvo ausente en todos los puntos.

\section{Discusión.}

Los resultados mostrados evidencian que este ecosistema está siendo alterado, ya que en varios meses del año todos los indicadores se observaron muy por encima del LMP. Si bien es cierto en la actualidad no se consideran a los coliformes totales como buenos indicadores de contaminación, estos se tomaron en cuenta ya que al momento del estudio estaban considerados dentro de los parámetros de los ECAs (ECAs, 2008). Este incremento se debe, principalmente, a la interacción que tiene la población con el humedal. En un futuro, estos impactos podrían ocasionar cambios en la composición, estructura y dinámica del ecosistema, convirtiéndolo en una potencial fuente para la proliferación de enfermedades.

Es importante considerar que no todos los coliformes totales representan una amenaza para la salud. Por ejemplo, los coliformes Serratia spp. y

Tabla 2. Concentración bacteriana de coliformes termotolerantes (NMP/100 mL) en la laguna "Pisciplaya" por punto de muestreo. Los valores sombreados corresponden a los valores que sobrepasan los LMP.

\begin{tabular}{|c|c|c|c|c|c|c|c|c|c|c|c|c|c|c|c|}
\hline \multirow[b]{2}{*}{ Mes } & \multicolumn{15}{|c|}{ Puntos de muestreo } \\
\hline & 1 & 2 & 3 & 4 & 5 & 6 & 7 & 8 & 9 & 10 & 11 & 12 & 13 & 14 & 15 \\
\hline Agosto & 45 & $<1.80$ & $<1.80$ & $<1.80$ & 410 & 790 & 110 & 40 & 1100 & 490 & 1300 & 1100 & 330 & 200 & $<1.80$ \\
\hline Septiembre & 45 & $<1.80$ & $<1.80$ & $<1.80$ & 410 & 790 & 110 & 40 & 1100 & 490 & 1300 & 1100 & 330 & 200 & $<1.80$ \\
\hline Octubre & 490 & 9200 & 1300 & 330 & 330 & 78 & 170 & 410 & 2400 & 3500 & 1100 & 790 & 170 & 45 & 78 \\
\hline Noviembre & 20 & 310 & 170 & 20 & 130 & 130 & $<1.80$ & 20 & 20 & 61 & 78 & 220 & $<1.80$ & 45 & $<1.80$ \\
\hline Diciembre & 1100 & 490 & 490 & 230 & 490 & 1700 & $<1.80$ & 20 & 20 & 45 & 78 & 220 & 78 & 1400 & 45 \\
\hline Enero & 490 & 1100 & 110 & 45 & 1100 & 230 & 1100 & 460 & 340 & 490 & 3500 & 330 & 330 & 790 & 20 \\
\hline Febrero & 330 & 1100 & 20 & 45 & 68 & 490 & 110 & 330 & 1300 & 1300 & 460 & 490 & 170 & 170 & 68 \\
\hline Marzo & 170 & 270 & 20 & 78 & 110 & 170 & 68 & 230 & 1700 & 1300 & 230 & 220 & 220 & 210 & 68 \\
\hline Abril & 130 & 220 & 20 & 110 & 110 & 220 & 68 & 230 & 460 & 330 & 230 & 220 & 130 & 170 & 45 \\
\hline Mayo & 45 & $<1.80$ & $<1.8$ & 20 & $<1.8$ & 40 & 130 & 140 & 20 & 490 & 940 & 130 & 61 & 170 & 40 \\
\hline Junio & 1700 & 790 & 490 & 230 & 790 & 140 & 40 & 170 & 130 & 100 & 230 & 790 & 110 & 390 & 45 \\
\hline Julio & 240 & 1301 & 790 & 230 & 1700 & 790 & 3500 & 5400 & 700 & 2800 & 5400 & 2800 & 490 & 220 & 790 \\
\hline PROMEDIO & 400.4 & 1231.8 & 284.2 & 111.5 & 512.5 & 464.0 & 450.5 & 624.2 & 774.2 & 949.7 & 1237.2 & 701.8 & 201.6 & 334.2 & 99.9 \\
\hline
\end{tabular}

Valor de referencia de LMP para coliformes termotolerantes $=1000 \mathrm{NMP} / 100 \mathrm{~mL}(\mathrm{ECAs}, 2008)$ 
Tabla 3. Concentración bacteriana de Escherichia coli (NMP/100 mL) en la laguna "Pisciplaya" por punto de muestreo. Los valores sombreados corresponden a los valores que no sobrepasaron los LMP.

\begin{tabular}{lrrrc}
\hline & \multicolumn{4}{c}{ Puntos de Muestreo } \\
\cline { 2 - 5 } Mes & \multicolumn{1}{c}{5} & \multicolumn{1}{c}{5} & 10 & 15 \\
\hline Agosto & 45 & 210 & 330 & $<1.80$ \\
Septiembre & 45 & 170 & 230 & $<1.80$ \\
Octubre & 230 & 170 & 2400 & 78 \\
Noviembre & 20 & 45 & $<1.80$ & $<1.80$ \\
Diciembre & 110 & 330 & 45 & 45 \\
Enero & 20 & 92 & 45 & $<1.80$ \\
Febrero & 170 & 20 & 340 & 20 \\
Marzo & 170 & 110 & 1300 & 680 \\
Abril & 130 & 20 & 170 & 20 \\
Mayo & 20 & $<1.80$ & 20 & $<1.80$ \\
Junio & 330 & 330 & 100 & 18 \\
Julio & 220 & 1300 & 1300 & 490 \\
\hline Valor
\end{tabular}

Valor de referencia de LMP para Escherichia coli $=$ Ausente NMP/100mL (ECAs, 2008).

Hafnia spp. pueden crecer en las lagunas dado que forman parte de este tipo de ecosistemas (Ashbolt et al., 2001). Sin embargo, en el presente estudio los altos valores de coliformes totales evidenciarían que hay una fuente rica de nutrientes que mantiene a esta comunidad bacteriana, posiblemente relacionada a los canales y desagües que llegan a la laguna.

Por otro lado, la presencia de los coliformes fecales en el ACR Humedales de Ventanilla representan un gran riesgo para la salud de la población que utiliza sus aguas como fuente recreativa y extractiva (recientemente se han introducido peces como Ciprinus carpio "carpa" con fines alimenticios (Carazas et al., 2015). Dentro de este grupo de microorganismos dañinos se considera a bacterias patógenas como los patoptipos de Escherichia coli, Klebsiella spp. y Enterobacter spp. que podrían provocar gastroenteritis (por ingestión), infecciones respiratorias, en los oídos, la piel y otras (Robert,

Tabla 4. Concentración bacteriana de Enterococcus faecalis (NMP/100 mL) en la laguna "Pisciplaya" por punto de muestreo. Los valores sombreados corresponden a los valores que no sobrepasaron los LMP.

\begin{tabular}{lrrrr}
\hline & \multicolumn{4}{c}{ Punto de Muestreo } \\
\cline { 2 - 5 } Mes & \multicolumn{1}{c}{5} & \multicolumn{1}{c}{5} & 10 & 15 \\
\hline Agosto & 1100 & 700 & 14000 & 400 \\
Septiembre & 230 & 330 & 470 & 45 \\
Octubre & 240 & 400 & 1700 & 20 \\
Noviembre & 40 & 360 & 61 & 680 \\
Diciembre & 790 & 490 & 380 & 93 \\
Enero & 400 & 1200 & 1700 & 78 \\
Febrero & 4300 & 2800 & 400 & 130 \\
Marzo & 3500 & 700 & 270 & 170 \\
Abril & 340 & 1200 & 2200 & 100 \\
Mayo & 130 & 270 & 330 & 78 \\
Junio & 130 & 260 & 170 & 170 \\
Julio & 110 & 200 & 230 & 20 \\
\hline Valor de referencia de LMP para Enterococcus faecalis $=200$ \\
NMP/100mL (ECAs, 2008).
\end{tabular}

2010).

En el análisis realizado en el presente trabajo se ha considerado la laguna como un espacio destinado a la conservación del ambiente acuático (categoría 4). Si consideramos el uso recreativo como parte intrínseca de la laguna, esta podría pasar a la categoría 1B1 (laguna recreativa de uso directo en donde la población la utiliza para bañarse y consume sus recursos). De ser así, este cuerpo de agua sobrepasaría también los LMP para Escherichia coli y Enterococcus faecalis no considerados en la categoría 4 (LMP <200 NMP/100 mL). La presencia de cargas altas de estas dos especies indica una contaminación con heces humanas y de animales o de cercanía a aguas residuales poniendo en peligro la salud de la población (Wheeler et al., 2002). De estas dos bacterias, Enterococcus faecalis indica contaminación fecal periódica y de largo tiempo, ya que esta especie no se multiplica en el medio ambiente (De Navia \& Estupiñán-Torres, 2006). Esta contaminación fecal pudo verificarse en la zona durante el monitoreo (una parte de las inmediaciones del humedal se encontraron de libre acceso por perros callejeros y de personas los cuales dejan sus desperdicios cerca a los cuerpos de agua o dentro de ella). Cerca al punto 10 existe un canal de aireación cuya función es permitir el movimiento de las aguas estancadas de la laguna pero que al momento del monitoreo se encontraba cargada con la presencia de basura doméstica. De esta forma, el canal pudo convertirse en uno de los puntos de origen de contaminación bacteriana de la laguna lo cual se refleja en las tablas 3 y 4, siendo esta estación la que tiene más alta concentración de estas bacterias.

Vibrio es un género que agrupa especies patógenas para el ser humano y los animales entre ellas la especie $V$. cholerae, agente etiológico de numerosas epidemias de cólera en el mundo. Otras especies de Vibrio están relacionadas a diarreas inflamatorias e infecciones cutáneas. (Cabrera, 2007) La presencia constante de Vibrio spp. en el humedal sugiere que puede tratarse de una reserva acuática asociadas a sedimentos u organismos acuáticos (Jiménez-Madrazo et al.,1994), por lo que el consumo de estos recursos podría provocar problemas serios de salud a la población de animales y seres humanos aledaña.

A pesar de que estos parámetros son utilizados como indicadores de la contaminación de cuerpos de agua, en el Perú el seguimiento en las lagunas de los humedales es pobre. Es necesario establecer la variabilidad temporal de la calidad de agua, ya que esto nos permite establecer el grado de contaminación del ecosistema acuífero durante el tiempo y poder determinar medidas de conservación por parte de las instituciones correspondientes. Se recomienda que los resultados del presente trabajo deban ser tomados en cuenta para la generación de herramientas de gestión, que eviten el uso de los cuerpos de agua con fines recreativos y extractivos para consumo humano, así 
Tabla 5. Presencia de Vibrio spp. en la laguna "Pisciplaya" por punto de muestreo. Los valores sombreados corresponden a los valores que no sobrepasaron los LMP.

\begin{tabular}{lcccc}
\hline & \multicolumn{5}{c}{ Puntos de Muestreo } \\
\cline { 2 - 5 } Mes & 1 & 5 & 10 & 15 \\
\hline Agosto & 1 & 1 & 1 & 0 \\
Septiembre & 1 & 1 & 1 & 1 \\
Octubre & 1 & 1 & 1 & 1 \\
Noviembre & 1 & 1 & 1 & 1 \\
Diciembre & 1 & 1 & 0 & 0 \\
Enero & 1 & 1 & 1 & 0 \\
Febrero & 1 & 1 & 1 & 0 \\
Marzo & 1 & 1 & 1 & 1 \\
Abril & 1 & 1 & 1 & 1 \\
Mayo & 1 & 1 & 1 & 1 \\
Junio & 1 & 1 & 0 & 0 \\
Julio & 1 & 1 & 1 & 0 \\
\hline 1=Presente; 0=Ausente. & & & \\
Valor de referencia de LMP & para Vibrio & spp. \\
Ausencia/100mL (ECAs, 2008). & &
\end{tabular}

como la contaminación de los canales que alimentan al humedal.

\section{Agradecimientos.}

Los autores quedamos profundamente agradecidos con la Gerencia de Medio Ambiente del Gobierno Regional del Callao por habernos brindado las facilidades para poder realizar el monitoreo.

\section{Literatura citada.}

Alvarez C. \& Iannacone J. 2008. Nuevos registros de aves en los humedales de Ventanilla, Callao, Perú. Biologist (Lima). 6 (1): 68-71.

Aponte H. \& Cano A. 2013. Estudio florístico comparativo de seis humedales de la costa central del Perú: Actualización y nuevos retos para su conservación. Revista Latinoamericana de Conservación. 3(2): 15-27.

Aponte H. \& Ramirez D.W. 2014. Riqueza florística y estado de conservación del Área de Conservación Regional Humedales de Ventanilla (Callao, Perú). Biologist (Lima). 12(2): 270-282.

Aponte H., Ramírez D., Lértora G., Vargas R., Gil F., Carazas N. \& Liviac R. 2015 Incendios en los Humedales de la Costa Central del Perú: Una amenaza frecuente. Científica. 12(1): 70-81.

Ashbolt N.J., Grabow W.O.K. \& Snozi M. 2001 Indicators of microbial water quality. En: Water quality: Guidelines, standards and health - Assessment of risk and risk management for water-related infectious disease Water Series (eds. L. Fewtrell \& Bartram). IWA Publishing, Londres, UK. pp. 289-315.

Luis E. Cabrera R., Graciela Castro E., M. Margarita Ramírez A., Alina Llop H., Rafael Llanes C., Neisideismy Castañeda E., Anabel Fernández A. \& Laura Bravo F. 2007 Aislamiento e identificación de especies pertenecientes a los géneros Aeromonas, Vibrio y Plesiomonas procedentes de muestras extra- intestinales en Cuba. Rev. chil. infectol. 24(3): 204-208. http://dx.doi.org/10.4067/S0716-10182007000300005.

Carazas N., Gil F., Liviac R., Zárate R. \& Montalvo J. 2015. Nuevos Registros de Fauna en el Área de Conservación Regional (ACR) Humedales de Ventanilla. Cientifica. 12(1): 42-60.

De Navia S.L.Á. \& Estupiñán-Torres S.M. 2006. Bacteriological quality of the water of the humedal of Jaboque, Bogotá, Colombia. Caldasia. 28: 67-78.

Gobierno Regional del Callao. 2009. Plan Maestro 2009 2014 Del Área de Conservación Regional Humedales de Ventanilla. Gobierno Regional del Callao.

Guillén G., Aponte H., Bacigalupo X. \& Rodriguez R. 2015. Protozoarios de vida libre del Área de Conservación Regional Humedales de Ventanilla (Callao - Perú) en el período septiembre 2011 - enero 2012. Científica. 12(1): 61-69.

Iannacone J., Atasi M., Bocanegra T., Camacho M., Montes A., Santos S., Zuñiga H. \& Alayo M. 2010. Diversidad de aves en el humedal Pantanos de Villa, Lima, Perú: periodo 2004-2007. Biota Neotropica. 10: 295-304.

Jiménez-Madrazo C., Haro-Ramor R.A., Lázaro de la Escozura J.L. \& Móntes-Gómez J.E. 1994. Asilamiento ambiental de Vibrio Cholerae O1 en aguas continentales de la provincia de Sevilla. Revista Española de Salud Pública. 68(1): 187-196.

León B., Cano A. \& Young K. 1998. Uso Actual de la Flora y Vegetación en los Humedales de la Costa Central del Perú. En: Los Pantanos de Villa: Biología y Conservación Serie de Divulgación $\mathrm{N}^{\circ} 11$. (eds A. Cano \& K. Young), Universidad Nacional Mayor de San Marcos, Lima - Perú. pp. 191-104.

Pullés M.L. 2014. Microorganismos indicadores de la calidad del agua potable en Cuba. Revista CENIC Ciencias Biológicas. 45(1): 25-36.

Rice E.W., Bridgewater L., Eaton A. \& Clesceri L. 2012. Standard Methods for the Examination of Water and Wastewater, 22nd ed. American Public Health Association, USA.

Riveros J., Pautrat L. \& Gushiken S. 1998. Bases de un Plan Ecoturístico para los Pantanos de Villa. En: Los Pantanos de Villa: Biología y Conservación Serie de Divulgación $\mathrm{N}^{\circ} 11$. (eds K. Young \& A. Cano). Universidad Nacional Mayor de San Marcos, Lima Perú. pp. 133-146.

Robert Pullés M. 2014 Microorganismos indicadores de la calidad del agua potable en cuba. Revista CENIC. Ciencias Biológicas. 45(1): 25-36. Disponible en: http://www.redalyc.org/articulo.oa?id=181230079005. Fecha de consulta: 3 de enero de 2017.

Rojas Rieckhof, M.A. 2010. Situación actual y perspectivas turísticas de los Humedales de Ventanilla - Callao. CULTURA: Lima (Perú). 24: 1-20.

Wheeler A.L., Hartel P.G., Godfrey D.G., Hill J.L. \& Segars W.I. 2002. Potential of Enterococcus faecalis as a human fecal indicator for microbial source tracking. Journal of Environmental Quality. 31: 1286-1293.

Young K. 1998. El Ecosistema. En: Los Pantanos de Villa: Biología y Conservación (eds A. Cano \& K. Young). Universidad Nacional Mayor de San Marcos, Lima Perú. pp. 3-20. 
1 Área de Biología, Coordinación Cursos Básicos. Universidad Científica del Sur. Av. Antigua Carretera Panamericana Sur km 19 Villa El Salvador. - Lima 42, Perú. Email: rovi81@hotmail.com, rocio_retach@hotmail.com.

${ }^{2}$ Instituto de Genómica y Terapias Avanzadas Xecuenxia Av. Brasil 3615 Magdalena del mar. - Lima 17, Perú.

${ }^{3}$ Escuela de Biología Marina, Facultad de Ciencias Biológicas y Veterinarias. Universidad Científica del Sur. Av. Antigua Carretera Panamericana Sur km 19 Villa El Salvador. - Lima 42, Perú.

${ }^{4}$ Laboratorio de Florística, Museo de Historia Natural, Universidad Nacional Mayor de San Marcos. Avenida Arenales 1256, Jesús María - Lima. Apartado 14-0434, Lima 14, Perú.

${ }^{5}$ Laboratorio Clínico, Hospital Militar Central. Av. Faustino Sánchez Carrión s/n, Jesús María - Lima 42, Perú.

${ }^{6}$ Laboratorios de Microbiología. Facultad de Ciencias de la Salud. Universidad Científica del Sur. Av. Antigua Carretera Panamericana Sur km 19 Villa El Salvador. - Lima 42, Perú. 